\title{
Electronic Band Structure and Complex Dielectric Function of zb-AlP: A First Principles Study
}

\author{
K. Uma Mahendra Kumar
}

Dept of Physics, School of Advanced Science, Vellore Institute of Technology, Vellore-632014, India

(Received May 6, 2019; revised version June 13, 2019; in final form August 12, 2019)

From first principles, the electronic and optical properties of zb-AlP are calculated. Based on calculated band structure, within the RPA approximation, the complex dielectric function and linear optical properties are calculated. With a band gap of $1.54 \mathrm{eV}$, zb-AlP shows typical wide band gap semiconductor like optical properties. The plasmon frequency $\omega_{p}$ is found in the ultraviolet region.

DOI: 10.12693/APhysPolA.136.486

PACS/topics: aluminum phosphide, optical properties, DFT, complex dielectric function

\section{Introduction}

Aluminum phosphide (AlP) is well known semiconductor with wide band gap. With other binary compounds of III-V semiconductors AlP forms wide variety of semiconductor compositions and is used in electronic industry for light emitting device (LED) applications.

Apart from LED applications, AlP has become most probable dilute magnetic semiconducting (DMS) material in recent years. Compared with Mn-doped AlN, more stable ferromagnetic state has been found in Mn-doped AlP [1]. In a theoretical study of Katayama-Yoshida and Sato the Curie temperature of $5 \%$ Mn-doped AlP was above the room-temperature [2]. An enhanced optical absorption is also observed when AlP is doped with Mn [1]. Olsson et al. has investigated many more interesting properties of AlP when doped with $\mathrm{Cr}$ [3]. They have proposed that dilute $\mathrm{Cr}$ doped AlP can be an efficient photovoltaic material.

All the above mentioned merits are computationally attributed to AlP and AlP lacks experimental evidence. Very few experimental reports are available about the intrinsic optical properties of $\operatorname{AlP}[4,5]$. It is surprising that for a material like AlP, which has been extensively used in LED applications and found to be the most favorable semiconductor for DMS and photovoltaic applications, the detailed studies of the electronic and optical properties are seldom available.

In this present work, we investigate in detail the complex electric function and linear optical properties of zb-AlP using first principles. The derived data is interpreted with a fundamental interest in physics lying behind the spectral features in the complex dielectric function of zb-AlP.

corresponding author; e-mail: umamahendra@vit.ac.in

\section{Computational details}

The ground-state of zb-AlP is calculated using first principle total-energy based on density functional theory (DFT) as implemented in GPAW code [6]. The kinetic energy cut-off for the plane waves are expanded up to $450 \mathrm{eV}$. A uniform mesh of $13 \times 13 \times 13 k$-points are used to sample the Brillouin zone of zb-AlP.

Bulk model of zb-AlP was generated using atomic simulation environment (ASE) [7] with fundamental structural parameters taken from crystallographic open database with entry no. 9008831 [8]. AlP crystallizes in two different crystallographic forms: zinc-blende and wurzite. We have chosen the zinc-blende form for the current studies. The initial lattice parameter is $a=5.451 \AA$ with $\mathrm{Al}$ at $(0,0,0)$ and $\mathrm{P}$ at $(0.25,0.25,0.25)$. All the calculations were carried out within the generalized gradient approximation (GGA) for exchange-correlation energy [9]. The lattice parameter is optimized by following the standard procedure of fitting the energy vs. volume curve with the Murnaghan equation of state (EOS) [10].

The complex dielectric function, $\hat{\epsilon}(\boldsymbol{q}, \omega)=\epsilon_{1}+\mathrm{i} \epsilon_{2}$, is calculated from the ground state electronic structure, where $\boldsymbol{q}$ is transferred momentum, $\boldsymbol{\omega}$ is the frequency, $\epsilon_{1}$ is real part, and $\epsilon_{2}$ is the imaginary part of the dielectric function. For the above, a frequency dependent linear response function is calculated within the frame work of time-dependent density functional theory (TD-DFT) using projector augmented wave method. For the exchange and correlation energy, corrections are calculated using random phase approximation (RPA).

\section{Results and discussion}

At standard conditions aluminum $(\mathrm{Al})$ and phosphorus $(\mathrm{P})$ with their natural electronegativity and atomic size result in a compound semiconductor AlP, whose bond nature is covalent as well as ionic. As stated previously, AlP crystallizes in zinc-blende structure, with tetrahedral coordination. 
The structural properties of zb-AlP are detailed in Table I. The calculated lattice parameter $a_{0}$ is 5.5125 which is in good agreement with previous calculation of $a_{0}$ which is 5.52 [13], in contrast with the experimental lattice parameter $a_{0}$ as 5.4510 [8]. The estimated lattice parameter value is $1.12 \%$ deviated from the experimental value.

TABLE I

The optimized lattice parameter of zb-AlP compared with experimental values, where $a_{0}$ is lattice parameter, $B_{0}$ is bulk modulus

\begin{tabular}{l|c|c|c}
\hline \hline \multicolumn{1}{c|}{ zb-AlP } & $a_{0}[\AA]$ & $B_{0}[\mathrm{GPa}]$ & Volume $\left[\AA^{3}\right]$ \\
\hline GGA & 5.5125 & 82.10 & 167.51 \\
Exp. [9] & 5.4510 & - & 161.96 \\
Calc. [11, 12] & 5.480 & - & - \\
Calc. [8] & 5.52 & 86.5 & -
\end{tabular}

The bulk modulus, $B_{0}$ of zb-AlP is estimated as $82.10 \mathrm{GPa}$ in the present work. This value is in good agreement with previous calculation with $B_{0}$ of $86.5 \mathrm{GPa}$. Estimated structural parameters from GGA are in good agreement with experimental findings. All further calculations, such as electronic band structure and complex dielectric function are obtained within the GGA approximation.

\subsection{Electronic band structure}

The ground-state electronic band structure of zb-AlP is shown in Fig. 1. In Fig. 2, we have shown the total density of states (TDOS) in the part (a) and in $(b-c)$ we have shown the partial density of states (PDOS) of $\mathrm{Al}$ and $\mathrm{P}$ atom as sum over all bound states with the specific angular momentum.

The electronic bands are grouped into three different regions. The top of the valence band (VB) is set to zero on the energy scale marking the Fermi level.

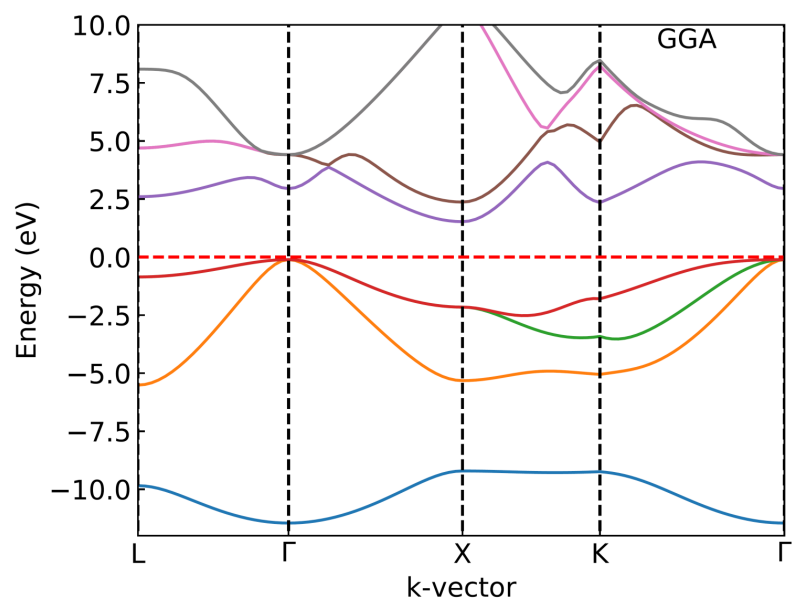

Fig. 1. Electronic band structure of zb-AlP calculated with in the GGA approximation.

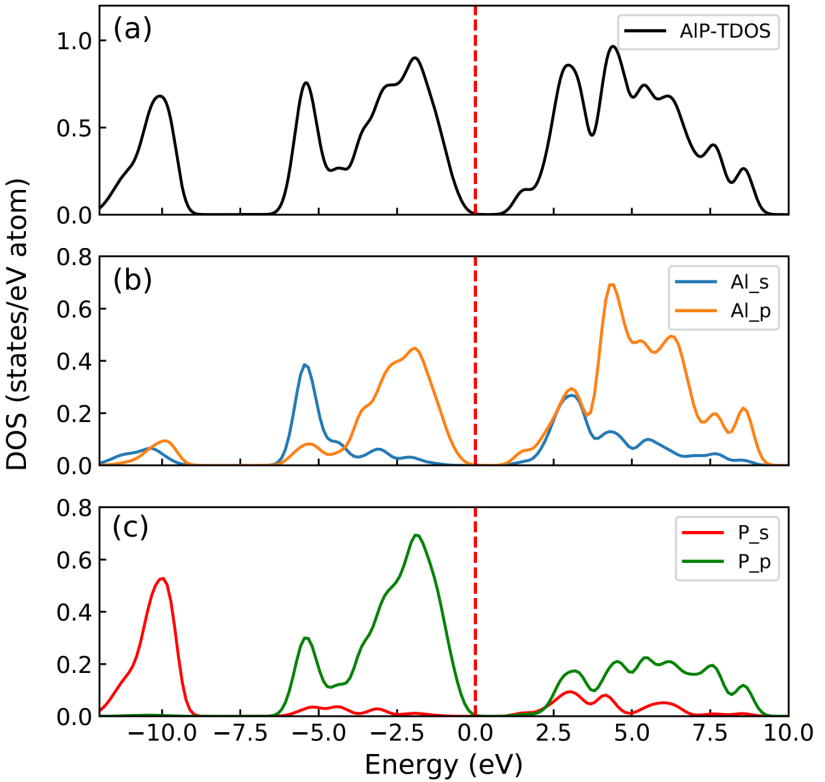

Fig. 2. Total and partial density of states (PDOS) of particular atom as sum over all bound states with the specific angular momentum; (a) total density of states (TDOS), (b) partial density of states of $\mathrm{Al}$ and (c) partial density of states of $\mathrm{P}$.

A group of four bands with small overlapping are spanned up to $10 \mathrm{eV}$ above the Fermi level. These bands have major contribution from $\mathrm{Al} 3 p$ states and $\mathrm{Al} 3 s$ states. Moreover, a small contribution from P $3 p$ and $3 s$-states is always present because of the covalent character of the Al-P bond. The same is clear from part (b) and (c) in Fig. 2. This set of bands forms the conduction band $(\mathrm{CB})$ in zb-AlP.

Below the Fermi level up to $-6 \mathrm{eV}$, there are three bands mostly contributed by aluminium and phosphorus $3 p$ states. Due to the $s p$-hybridization resulting from Al $3 s$-states and phosphorus $3 p$ states a peak emerges in the TDOS at around $-5.4 \mathrm{eV}$. Next, in the energy range of -8.0 to $-12 \mathrm{eV}$, this group of bands primarily comes from phosphorus $3 s$ states with small contribution from $\mathrm{Al} 3 s$-states. This set of bands forms the $\mathrm{VB}$ in zb-AlP.

Within the limitation of GGA approximation the estimated band gap is $1.54 \mathrm{eV}$, and it is an underestimation, when compared with experimental value 2.45 [14]. The nature of the band gap is indirect and occurs between high symmetry points $\Gamma$ and $\mathrm{X}$ in the first Brillouin zone, which is in agreement with the experiment.

\subsection{Complex dielectric function $\epsilon$}

Within the RPA approximation, the complex dielectric function is derived from the electronic band structure calculated under GGA. The spectral dependence of $\epsilon_{1}$ and $\epsilon_{2}$ is shown in Fig. 3a. The optical properties shown by zb-AlP are of a typical wide bandgap semiconductor. 


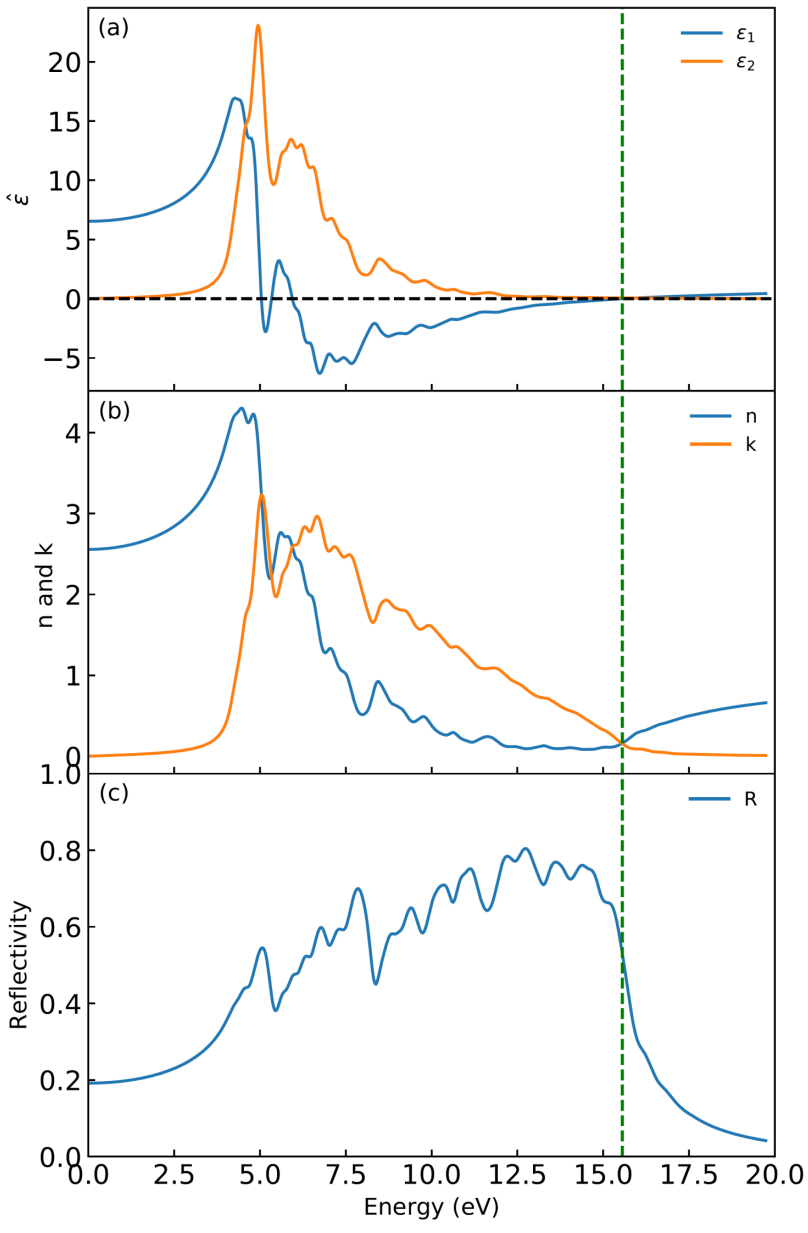

Fig. 3. Calculated spectral dependence of (a) $\epsilon_{1}$ and $\epsilon_{2}$ of zb-AlP, (b) refractive index $n$ and extinction coefficient $k$ and (c) the reflectivity calculated from Eq. (1). The dashed green line marks the plasmon frequency $\left(\omega_{p}\right.$ at $\left.15.56 \mathrm{eV}\right)$.

The real part of the dielectric function, $\epsilon_{1}$, is continuously increased till $4.3 \mathrm{eV}$, marking the normal dispersion region of zb-AlP. Then, up to $5 \mathrm{eV}, \epsilon_{1}$ decreases with energy and marks the anomalous dispersion region. Due to the inter-band transitions, between 5.26 to $5.91 \mathrm{eV}, \epsilon_{1}$ becomes positive with a spectral feature peaking at $5.33 \mathrm{eV}$ and crosses zero at $5.95 \mathrm{eV}$ to become negative. Further, $\epsilon_{1}$ again continues to increase as a function of energy and becomes positive at $15.56 \mathrm{eV}$. The peak maximum of the imaginary part $\epsilon_{2}$, represents the inflection point in $\epsilon_{1}$. For zb-AlP this inflection point is located at $4.95 \mathrm{eV}$. The shoulder peak on the $\epsilon_{2}$ is due to interband transitions.

As shown in Fig. 3b, the refractive index $n$ and extinction coefficient $k$ are plotted against the energy. At the limit $\omega \rightarrow 0$ the $\epsilon_{1}$ is approximately 6.5 and as a result, the refractive index $n=2.55$. These results are in excellent agreement with the optical properties derived from spectroscopic ellipsometry [5]. With an intermediate range of refractive index, zb-AlP can have small amount of reflectivity (see Fig. 2c) with no appreciable absorption and more transparency in low energy region. Further, reflectivity increases from 5.0 to $15 \mathrm{eV}$, then gradually decreases, marking the plasmon frequency in the ultra violet region $\left(\omega_{p}\right.$ at $\left.15.56 \mathrm{eV}\right)$. Apart from that, around 5.2 and $8.2 \mathrm{eV}$, zb-AlP has strong interband transitions, which are markedly visible in $\epsilon_{1}$ also as distinct peaks.

In normal incidence conditions the reflectivity can be calculated from the following relationships [15]:

$$
R=\frac{(1-n)^{2}+k^{2}}{(1+n)^{2}+k^{2}}
$$

where

$$
\begin{aligned}
& n=\left\{\frac{1}{2}\left[\left(\epsilon_{1}^{2}+\epsilon_{2}^{2}\right)^{1 / 2}+\epsilon_{1}\right]\right\}^{1 / 2}, \\
& k=\left\{\frac{1}{2}\left[\left(\epsilon_{1}^{2}+\epsilon_{2}^{2}\right)^{1 / 2}-\epsilon_{1}\right]\right\}^{1 / 2} .
\end{aligned}
$$

Here, $R$ is reflectivity, $n$ is refractive index, and $k$ is extinction coefficient. The reflectivity for zb-AlP is calculated using the $\epsilon_{1}$ and $\epsilon_{2}$ shown in Fig. 3c.

\section{Conclusions}

We have presented an investigation of linear optical properties of zb-aluminum phosphide based on first principle calculations. zb-AlP shows typical wide band gap semiconductor kind of reflectance with an indirect band gap of $1.54 \mathrm{eV}$. The electronic band structure is detailed in the light of the atom projected density of states (PDOS). The spectral dependence of dielectric function and linear optical properties like refractive index and extinction coefficient are depicted in detail.

\section{References}

[1] Y. Chen, Y. Xing, A. Jiang, C. Zhou, S. Lu, J. Magn. Magn. Mater. 457, 13 (2018).

[2] H. Katayama-Yoshida, K. Sato, J. Phys. Chem. Solids 64, 1447 (2003).

[3] P. Olsson, C. Domain, J.F. Guillemoles, Phys. Rev. Lett. 102, 227 (2009).

[4] Y.W. Jung, J.S. Byun, S.Y. Hwang, Y.D. Kim, S.H. Shin, J.D. Song, Thin Solid Films 519, 8027 (2011).

[5] S.Y. Hwang, T.J. Kim, Y.W. Jung, N.S. Barange, H.G. Park, J.Y. Kim, Y.R. Kang, Y.D. Kim, S.H. Shin, J.D. Song, C.T. Liang, Y.C. Chang, J. Alloys Comp. 587, 361 (2014).

[6] J.J. Mortensen, L.B. Hansen, K.W. Jacobsen, Phys. Rev. B 71, 035109 (2005).

[7] A.H. Larsen, J.J. Mortensen, J. Blomqvist, et al., J. Phys. Condens. Matter 29, 273002 (2017).

[8] R.W.G. Wyckoff, Crystal Structures, 2nd ed., Vol. 1, Interscience Publ., New York 1963, p. 85.

[9] J.P. Perdew, K. Burke, M. Ernzerhof, Phys. Rev. Lett. 77, 3865 (1996). 
[10] F.D. Murnaghan, Proc. Natl. Acad. Sci. USA, 30, 244 (1944).

[11] P. Rodriguez-Hernandez, A. Muhoz, Semicond. Sci. Technol. 7, 1437 (1992).

[12] C.O. Rodriguez, R.A. Casali, E.L. Peltzer, O.M. Cappannini, M. Methfessel, Phys. Rev. B 40, 3975 (1989).
[13] S. Lakel, F. Okbi, M. Ibrir, K. Almi, AIP Conf. Proc. 1653, 020065 (2015).

[14] M.R. Lorenz, R. Chicotka, G.D. Pettit, P.J. Dean, Solid State Commun. 8, 693 (1970).

[15] F. Wooten, Optical Properties of Solids, Academic Press, New York 1972. 